\title{
Distributed Localization Algorithm for Large-Scale Wireless Sensor Network
}

\author{
Hong Zhang \\ Weifang University, Weifang 261061, China \\ zhanghongwf@126.com
}

\begin{abstract}
Aiming at the inherent deficiencies of large-scale wireless sensor network for distributed positioning algorithm, the DV-hop algorithm is mainly studied and improved, and two kinds of improved algorithms DV-hop and DV-hop+Lastdist DV-hop-MinMax are proposed. The DV-hop+Lastdist improves the positioning accuracy of the node by only adding a message variable, while the DV-hop-MinMax effectively reduces the costs of floating-point operations and power consumption, and can select the appropriate algorithm according to actual application environment. Finally, the paper gives the method to realize OMNeT++ in the simulation environment, and carries out the simulation analysis.
\end{abstract}

Keywords: Wireless sensor networks, Distributed localization models, Node positioning algorithm, optimized algorithms

\section{Introduction}

The wireless sensor network with very broad work area applies to inaccessible poor or special environments. The sensor nodes are randomly scattered in the monitored area mainly through the aircraft sowing, manual embedding and rocket ejection. The location information of a node is random, and the data collected by the node are without application values if there is no location information. Therefore, in the application of wireless sensor network, node positioning has become a key issue. Currently, the node positioning algorithm of available wireless sensor network has common problems, such as environmental-impact ranging method, complex algorithm and high energy consumption, and inapplicability to the mobile node positioning [1]. Especially for large scale wireless sensor network, the node positioning algorithm is more prominently unpractical. This paper carries out discussions on the positioning algorithm applicable to large-scale wireless sensor network and puts forward improved algorithms.

\section{General Model for Fully Distributed Positioning Algorithm}

Based on the existing positioning algorithms for wireless sensor network, the paper selects three algorithms that meet basic requirements of the self-organization, robustness, and energy efficiency: Ad-hoc positioning [2], N-hop [3], and Robust positioning [4]. These three algorithms are all fully distributed, and are in direct broadcast communication with adjacent nodes, independent of any routing algorithm or any other ancillary infrastructure. Through the analysis of the three algorithms, a general model for fully distributed positioning algorithm which is applicable to large-scale wireless sensor network can be abstracted from.

\subsection{Distance Measurement between Unknown Node and Anchor Node}

In this stage, the distance between the unknown node and anchor node is determined for the location calculation of the second stage. Although the algorithms listed in this 
stage uses a different method, but they use the same communication mode: the anchor node initiates flooding communication, and broadcasts its location information and the corresponding distance information in the whole network.

2.1.1. DV-distance Algorithm: It is the simplest way to determine the distance between the unknown node and the anchor node by adding distance information to the each hop communication of flooding. The very method is used by DV-distance algorithm of Adhoc positioning to obtain the distance between a node and the unknown node [5,6]. In the DV-distance method for measuring point to point distance between adjacent nodes, first the anchor node sends a message containing its own identity and location information, and then each node receiving the message will add the measured length to the path length, and it will forward the message when the flooding limits allows. Another consideration is if a node receives the information of the previous anchor node from different paths, the path length will be updated and forwarded only when the current path length is less than the previous one. The final result is that each node stores the location information of a certain number of anchor nodes and the shortest path distance from the anchor node, preparing for the location calculation of the next stage.

2.1.2. DV-hop Algorithm: DV-distance algorithm has a drawback that the cumulative effect of the node ranging error [7] will occur during the multi-hop transmission. This cumulative error is more obvious when the network scale is large or anchor nodes are relatively few and node ranging hardware error is relatively large. A method with more robustness is to use the network topology information, and complete by calculating of hop count other than cumulative distance. This method is the very DV-hop algorithm. One of the biggest disadvantages of DV-hop algorithm is too dependent on hop location. When the anchor node can not receive a reasonable hop value for various reasons, it will largely affect the average distance value for each hop calculated by the anchor node; it is more complex for non-anchor nodes, they will receive unreasonable values and average distance value for each hop. Whatever the circumstances, the resulted positioning error will be enormous. Therefore, this paper proposes the Euclidean algorithm.

2.1.3. Euclidean Algorithm: First, the algorithm begins with that anchor node first broadcasts a beacon signal containing its own ID and location, and the TTL threshold of the signal is set to 2, that is, the beacon message can only be sent for two hops. When an unknown node can receive the signal from two nodes knowing mutual distance and the distance from the anchor node, it can calculate its distance from the anchor node through RSSI technology [8]. After an unknown node obtains the distances between three or more anchor nodes, it can localize itself.

\subsection{Node Positioning Calculation Coordinates}

In this stage, the node determines its own position based on the distance information of a certain number of anchor nodes provided by the three methods in the first stage.

2.2.1. Multilateral Measurement and Least Square Method: In the actual coordinate solving, multilateral measurement method is often used, which is the deformation of trilateration. Assume that the coordinates of known anchor nodes 1, 2, $3 \ldots n$ are $\left(x_{1}, y_{1}\right)$, $\left(x_{2}, y_{2}\right) \ldots\left(x_{n}, y_{n}\right)$, and their distance between the node $D$ are $d_{1}, d_{2}, \ldots, d_{n}$, respectively. Assume that the coordinate of the node $D$ is $(x, y)$, then the following formula is obtained:

$$
\left\{\begin{array}{l}
\left(x_{1}-x\right)^{2}+\left(y_{1}-y\right)^{2}=d_{1}^{2} \\
\vdots
\end{array}\right.
$$




$$
\left(x_{n}-x\right)^{2}+\left(y_{n}-y\right)^{2}=d_{n}^{2}
$$

The system of equations above can be linearized into the following forms by subtracting the nth equation:

$$
\begin{aligned}
& \left\{\begin{array}{l}
x_{1}^{2}-x_{n}^{2}-2\left(x_{1}-x_{n}\right) x+y_{1}^{2}-y_{n}^{2}-2\left(y_{1}-y_{n}\right) y=d_{1}^{2}-d_{n}^{2} \\
\vdots
\end{array}\right. \\
& x_{n-1}^{2}-x_{n}^{2}-2\left(x_{n-1}-x_{n}\right) x+y_{n-1}^{2}-y_{n}^{2}-2\left(y_{n-1}-y_{n}\right) y=d_{n-1}^{2}-d_{n}^{2}
\end{aligned}
$$

The readjusted system of equations can be expressed as $A x=b$.

2.2.2. Min-Max Method (Min-Max): Methods such as Multilateral measurement described above can still achieve very high positioning accuracy in the presence of certain error range, but the drawback is that these methods need for more floating point arithmetic, energy consumption caused by the computational expenditure still cannot be ignored, so it is necessary to seek a more simple replacement algorithm [9]. Min-Max is a relatively simple method, its basic idea is based on the unknown node to the reference node distance measurement and coordinate to construct several limit box, i.e., take the reference node as the center of the circle and take the unknown node to the reference node distance measurement value as radius circle to form the circumscribed square; Take intersection region of the square regarding the geometric center of the intersection region for the estimation coordinates of the unknown node, as shown in Figure 1. This figure illustrates the working principle of the three anchor node estimated distance, the node position is very close obtained by method of Min-Max and trilateration method, such as the intersection of three circles.

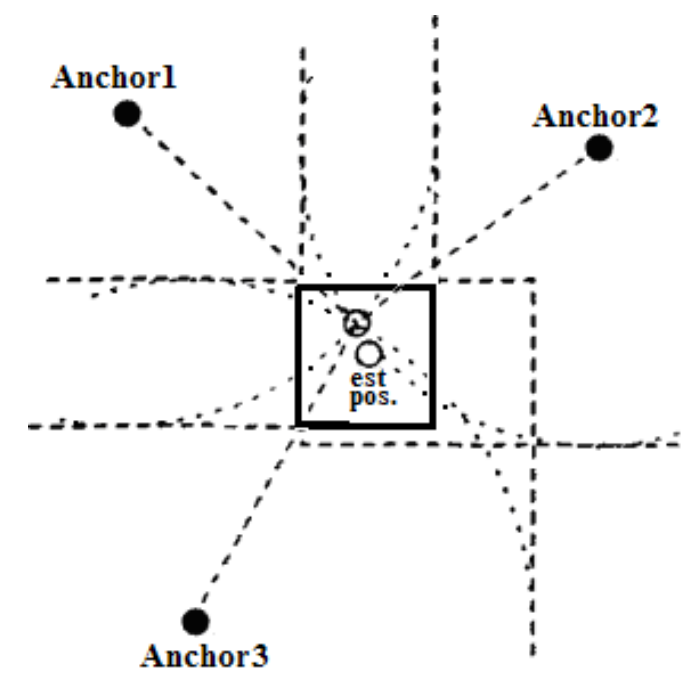

Figure 1. Min-Max Positioning Schematic Diagram

Boundary box of Anchor nodes $\mathrm{A}\left(x_{\mathrm{a}}, y_{\mathrm{a}}\right)$ is obtained by estimated distance $d_{\mathrm{a}}$ adding and subtraction: $\left[x_{\mathrm{a}}-d_{\mathrm{a}}, y_{\mathrm{a}}-d_{\mathrm{a}}\right] \times\left[x_{\mathrm{a}}+d_{\mathrm{a}}, y_{\mathrm{a}}+d_{\mathrm{a}}\right]$.

Intersection region boundary is obtained by taking the maximum among smaller values and minimum among larger values of all anchor nodes boundary box: $\left[\max \left(x_{\mathrm{i}}-d_{\mathrm{i}}\right), \max \left(y_{\mathrm{i}}-\right.\right.$ $\left.\left.d_{\mathrm{i}}\right)\right] \times\left[\min \left(x_{\mathrm{i}}+d_{\mathrm{i}}\right), \min \left(y_{\mathrm{i}}+\mathrm{d}_{\mathrm{i}}\right)\right]$. 
The final estimated value of unknown node coordinate is the intersection of geometric center. Min-Max method can get good results under the condition of without a large number of calculations [10].

\subsection{Iterative Refinement}

The purpose of this stage is to further refine and optimize the node position derived from the second stage. In the second stage, the distance from the anchor node is mainly used to estimate its location, and its distance relationship between adjacent nodes is not taken full advantage of. In this stage, the node will use the distance relationship between adjacent nodes to iterate and optimize the estimated location from the second stage. In this stage, the node first broadcasts its estimated position information, and receives the location information and the estimated distance of adjacent nodes, and to calculate its new location by running the positioning algorithm of the second stage. After repeated iterations, the node location information tends to be stabilized, and then refinement process is terminated, and the final position value is returned.

\section{Improved DV-hop Positioning Algorithm}

\subsection{Improved DV-hop Algorithm DV-hop+Lastdist}

Through subsequent simulation analysis we can see that the DV-hop is an extremely stable and predictable method. Because it does not need to use a radio signal measurement, it has no reaction to the ranging error parameter. But usually final jump of unknown nodes is less than the actual average distance, this leads to a slight overestimation of the distance between the node to anchor nodes, as shown in Figure 2. Node $\mathrm{A}$ is the anchor node, the circle for the node communication range, Node B, C, D for unknown nodes. Anchor nodes A sends a message to all nodes within communication range, and forward the message to the other nodes outside the communication range, because the nodes always search the least path from the anchor node hop, Node D by Node B and C forward to obtain the anchor node hop, but the CD itself are very close, their distance may be far less than the average distance of each jump, so when using the hop by the average jump distance to calculate the distance with the anchor node will cause greater error. In the higher percentage of anchor nodes and network connectivity, the needed hop from anchor node to unknown node will become less, the error will be more obvious [11].

In order to solve the distance overestimation caused by the final hop and maintain the high stability of the DV-hop, a distance variable of the final hop can be added to the message, and then the inherent ranging capacity of the node is used to obtain the final hop, while the former several hops are still calculated by using DV-hop. Therefore, now the distance from the unknown node to the anchor node is no longer the hop multiplied by the average distance per hop, but is calculated by using the following formula:

$$
\text { Distance }=(\text { hop count }-1) \times \text { average distance per hop }+ \text { final hop distance }
$$

By using this calculation method, the distance from the node to the anchor node can properly reduce the error distance caused by the final hop, so the method can also be called as DV-hop+Lastdist method. 


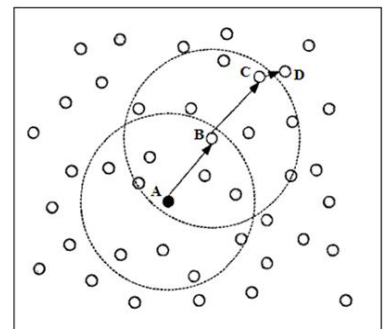

Figure 2. DV-hop Last Jump Distance Schematic Diagram

\subsection{Combination Method of DV-hop and Min-Max}

After the above discussion, we can see that multilateral measurement least-square method can still achieve very high positioning accuracy in the presence of certain error range. However, its drawback is that it needs more floating point arithmetic, the computational expenditure of energy consumption still cannot be ignored [12], the Min Max is a relatively simple calculation method, without involving too many matrix and floating point arithmetic, so in the case of strict energy consumption requirement, Min Max will have a big advantage than multilateral measurement method.

The traditional DV-hop with uses multilateral measurement least square method, which requires large costs of floating-point operations, so Min-Max method can be introduced into the DV-hop algorithm. In the first stage, the DV-hop method is used to calculate the distance between the unknown node and anchor node. In the second stage, the Min-Max method is used instead of the multilateral measurement least square method to calculate the node position as well as save the cost of floating point calculations. In the third stage, the traditional refinement method is still used, and finally a new DV-hop-MinMax method forms.

\section{Simulation and Result Analysis}

In order to test whether various location algorithms reach expected goal, the simulation tool OMNeT++ [13] is used. During the simulation test, the positioning error conditions of various algorithms in different scenarios are shown, but also the analysis is made on the influence of different network environment parameters on the network positioning performance.

\subsection{Establishment of Standard Scene}

At the beginning of simulation, nodes are randomly placed in a given square area by using random network topology generation method, and the network connectivity is controlled by the radio range of the node. At the end of the simulation, the corresponding simulation data are output such as node positioning error, and are ultimately presented by the average positioning error of all nodes, and the unsuccessful positioning nodes are not included in the calculation of the error average. To compare the positioning results under the different parameters, the ranging error and positioning error are normalized as values relative to the radio range. In the following simulations, the test is carried out based on the same standard scene and then makes comparison by changing different parameter values of the same standard scene.

\subsection{Analysis of Simulation Results}

The paper conducts simulation analysis on the node positioning based on using DV-hop, DV-distance and Euclidean algorithm in the standard scene with separate changes of parameters such as the ranging error, radio range, and the proportion of anchor node, some achievements in relative positioning error are obtained by three 
original algorithms under different conditions [14]. Similarly, the paper also conducts simulation analysis on the node positioning based on using improved DVhop algorithm in the standard scene with separate changes of parameters such as the ranging error, radio range, and the proportion of anchor node. Figure 1, Figure 2 and Figure 3 show the relative positioning error change curves of DV-hop algorithm and improved DV-hop algorithm in different conditions.

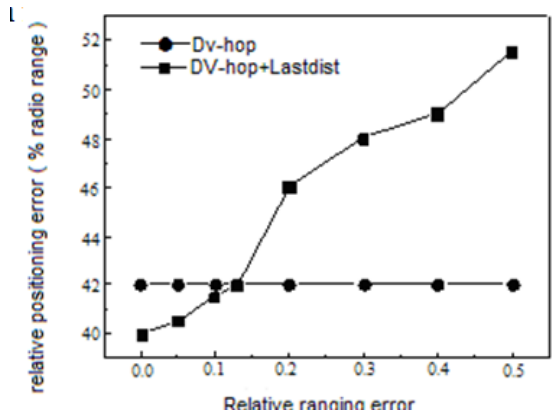

Figure 3. Curve of Relative Positioning Error of DV-hop+Lastdist with Changing Ranging Error

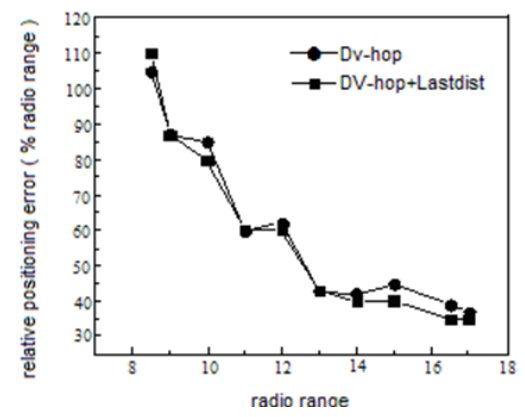

Figure 4. Curve of Relative Position Error of DV-hop+Lastdist with Changing Radio Range

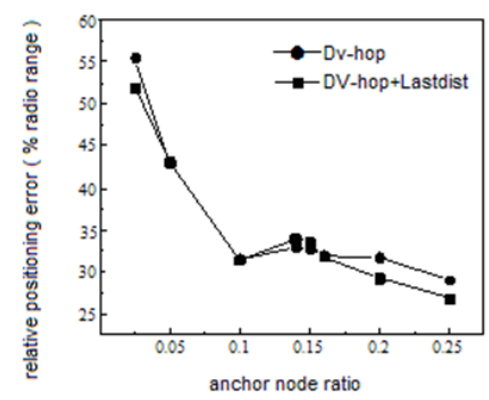

Figure 5. Curve of Relative Positioning Error of DV-hop+Lastdist with The Changing Ratio of Anchor Nodes

It can be seen from Figure 3 that although DV-hop+Lastdist is not as immune to the ranging error as DV-hop, but also the positioning accuracy show few fluctuations with the changing ranging error. When the ranging error is changing, generally the difference between the positioning errors of above two algorithms is small. But if the relative ranging error is less than 0.15, DV-hop+Lastdist will be better than DV-hop algorithm, while DV-hop is better than DV-hop+Lastdist in other cases. It can be seen from Figure 4 that when the radio range is small, that is, the connectivity is small, the performance of two algorithms are similar. But when the connectivity increases, DV-hop+Lastdist has a smaller relative positioning error than DV-hop. It can be seen from Figure 5 that when the 
anchor node ratio is low or high, especially when the ratio is high, the hop count from anchor nodes to unknown nodes may be smaller, and then DV-hop+Lastdist will be better than DV-hop. So when the node ranging error is small, network node connectivity or anchor node ratio is high, the DV-hop+Lastdist algorithm shall be used.

Figure 6 and Figure 7 and Figure 8 shows the Curve of relative positioning error of a new algorithm combining Dv-hop with Min - Max on the ranging error, radio range, and changes in the anchor node proportion.

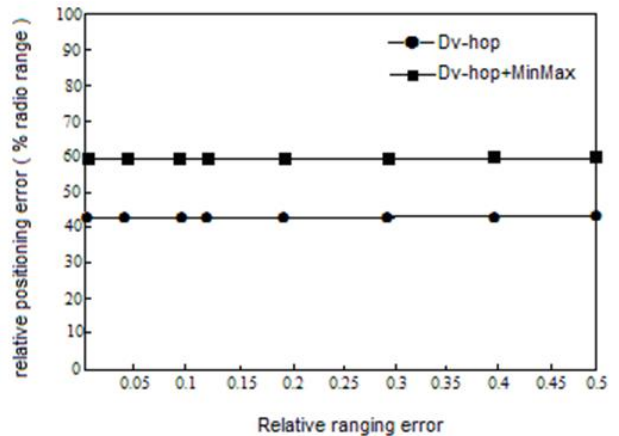

Figure 6. Curve of Relative Positioning Error of Dv-hop+MinMax Changing with Distance Measurement Error

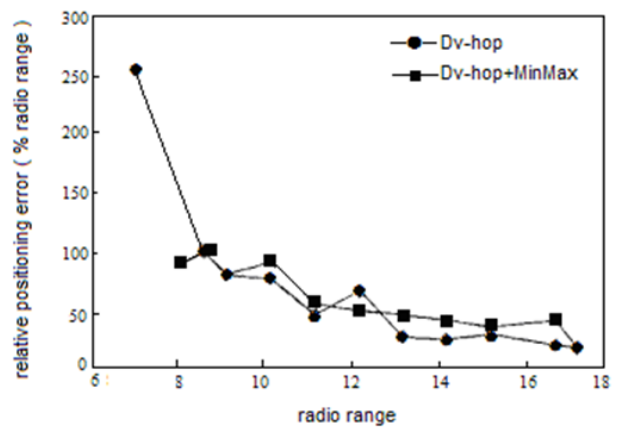

Figure 7. Curve of Dv-hop+MinMax Relative Positioning Error Changing with the Radio Range

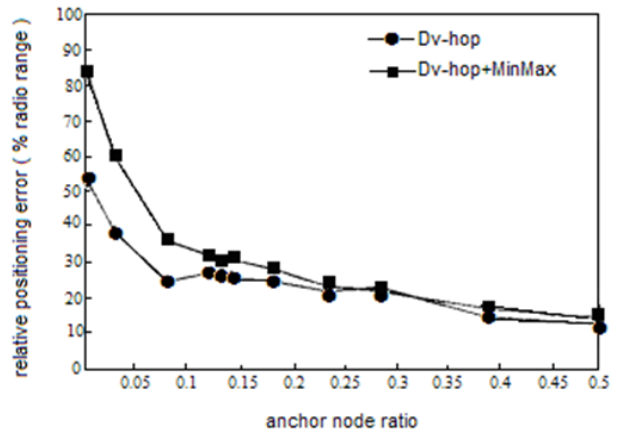

Figure 8. Curve of Dv-hop+MinMax Relative Positioning Error Changing with Anchor Node Proportion

From the above simulation results, we can see in most cases, the original Dv-hop has relatively lower positioning error than Dv-hop algorithm combined with Min - Max algorithm, in addition to the obvious difference under the standard scenario, when the other parameters change, the result is very approximate. However, we can see from Figure 7, when the network connectivity reduces to a very low condition, the traditional Dv-hop performance will worsen sharply, and the new algorithm still keeps quite gentle 
downward trend [15]. Figure 8 shows, when the anchor node proportion value is larger, the results error of two algorithms have been basically no different, considering the Dvhop+MinMax uses Min-Max algorithm to compute node position, this will save considerable floating-point computation expenditure, the new algorithm has a very big advantage undoubtedly. So when the network node connectivity is larger, and the propotion of anchor nodes is higher, it is easy to choose Dv-hop+MinMax algorithm.

\section{Conclusion}

Based on the analysis of principles of various positioning algorithms and the obtained simulation results, it can be shown that no algorithm can be optimal in all scenes, and the quality and choice of an algorithm depend on the ranging error, network connectivity, the ratio of anchor nodes, and the state of various network parameters. Therefore, the corresponding appropriate algorithm shall be selected depending on the applications. Even if the performance of an algorithm is more prominent on some occasions, it shall be also noted that the positioning accuracy of the algorithm still has much space to be improved.

\section{References}

[1] I. F. Akyildiz, W. Su, Y. Sankarasubramaniam and E. Cayirci, "Wireless sensor network, a survey", Computer Network, vol. 38, (2002), pp. 293-422.

[2] D. Nicolescu and B. Nath, "Ad-Hoc positioning systems (APS)", In: Proc, of the 2001 IEEE Global Telecommunications Conf., San Antonio, IEEE Communications Society, vol. 5, (2001), pp. 2926-2931.

[3] A. Sarrides, H. Park and M. B. Srirastara, "The bits and flops of the N-hop multilateration primitive for node localization problems", In: Prof, of the 1st ACM Int'1 Workshop on Wireless Sensor Networks and Applications. Atlanta: ACM Press, (2002), pp. 112-121.

[4] C. Savarese, J. Rabaey and K. Langendoen, "Robust positioning algorithms for distributed ad-boc wireless sensor networks", Proceedings of the General Track. 2002 USENLX Annual Technical Conference, Monterey, CA, USA, USENIX Assoc, (2002), pp. 317-327.

[5] D. Nicolescn and B. Nath, "Ad-boc Positioning Systems (APS)", In Proceedings of 2001 IEEE Global Telecommunications Conference (IEEE GLOBECOM'01), San Autonio, TX, USA: IEEE Communications Society, vol. 5, no. 11, (2001), pp. 2976-2986.

[6] D. Niculess and B. Nath, "DV based positioning in ad hoc networks", Journal of Telecommunication Systems, vol. 22, no. 1-4, (2003), pp. 267-280.

[7] J. Beutel, "Geolocation in a Pico Radio Environment ms Thesis", ETH Zurich Electronics Laboratory (1999), pp. 12.

[8] N. Bulusu, "Self-configuring Localization Systems", Ph. D. Thesis, University of California at Los Angeles, (2002), pp. 10.

[9] A. Varga, "The OMNeT++ discrete event simulation system, ASIM, European Simulation Multiconference (ESM-2001)", Prague, Czech Republic, SCS, (2001), pp. 319-325.

[10] J. J. Rabacy, M. J. Ammer, J. L. da Silva Jr., D. Patel, S. Roundy, "Picorodio supports ad hoe ultra-low power wireless networking", Computer, (2000), vol. 33, no. 7, pp. 42-48.

[11] C. Savarese, J. M. Rabaey and J. Beutel, "Locationing in distributed ad-hoc wireless sensor network", In Proc. of the 2001 IEEE Int' 1 Conf. on Acoustics, Speech, and Signal, vo1. 4, Salt Lake, IEEE signal Processing Society, (2001), pp. 2037-2040.

[12] S. CapKun, M. Hamdi and J. -P. Hubaux, "GPS-Free positioning in mobile ad-hoc networks", Cluster Computing, vol. 5, no. 2, (2002), pp. 157-167.

[13] L. Girod, V. Bychovskiy, J. EIson and D. Estrin, "Locating tiny sensors in time and space", a case study In Wemer B. ed. Proc. of the 2002 IEEE Int' 1 Conf. on Computer Design: VLSI in Computers and Processors. Freiburg: IEEE Computer Society, (2002), pp. 214-219.

[14] P. Bahl and V. N. Padmanabhan, "RADAR, An in-building RF-based user location and tracking system", In: Proc. of the IEEE INFOCOM 2000, Tel Aviv: IEEE Computer and Communications Societies, vo1. 2, (2000), pp. 775-784.

[15] B. Kobben, K. Van, A. Bunningen and K. Muthukrishnan, "Wireless campus LBS: Building campuswide location based services based on WiFi technology", Presented at the 1st intemational workshop on geographic hypermedia, Denver, U.S.A. Denver, ICA, (2005) April 4-5. 


\section{Author}

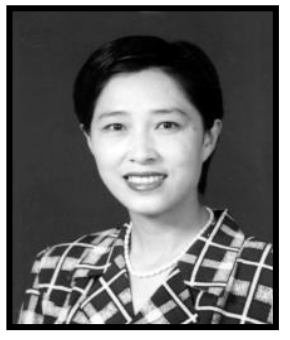

Hong Zhang, (1968) She is a professor, she graduated from Department of Electronic Engineering of Shandong University in 1991, she was majored in electronic information, obtaining a bachelor's degree in engineering, in 2006 she graduated from College of information and control engineering of Shandong University of Technology, obtaining master's degree in engineering. Her main research directions include intelligent control, network and communication. (E-mail: zhanghongwf@126.com) 
International Journal of Smart Home

Vol. 9, No. 10, (2015) 